\title{
Multi-fin kinematics and hydrodynamics in pufferfish steady swimming
}

\author{
Lijun Li ${ }^{1,2}$, Gen $\mathrm{Li}^{3}$, Ruoxin $\mathrm{Li}^{4}$, Qing Xiao ${ }^{4}$, and Hao Liu ${ }^{2,3}$ \\ ${ }^{1}$ School of Naval Architecture, Ocean and Civil Engineering, Shanghai Jiao Tong University, \\ 800 Dongchuan Road, Shanghai 200240, China \\ 2 Shanghai Jiao Tong University and Chiba University International Cooperative \\ Research Centre (SJTU-CU ICRC), Shanghai Jiao Tong University, China \\ ${ }^{3}$ Graduate School of Engineering, Chiba University, Japan \\ ${ }^{4}$ Department of Naval Architecture, Ocean and Marine Engineering, University of \\ Strathclyde, Glasgow, UK
}

\begin{abstract}
Pufferfish swim and maneuver with a multi-fin system including dorsal, anal, caudal, and pectoral fins, which presents sophisticated ventures in biomimetic designs of underwater vehicles. Distinguished from those 'typical' fish with streamlined body shape and body-caudal fin (BCF) undulations, pufferfish adopt non-streamlined plump body shape and rely on the oscillations and interplay of fins to achieve high performance maneuvering. Aiming at unveiling novel mechanisms associated with multi-fin kinematics and hydrodynamic performance in pufferfish swimming, we carried out an integrated study by combining measurement and digitizing of multi-fin kinematics and three-dimensional deformations and computational fluid dynamic (CFD) modeling of steady swimming. We constructed a realistic multi-fin kinematic model to mimic motions and deformations of the dorsal, anal, and caudal fins. We further built up a CFD model of the pufferfish with a realistic body and multi-fin geometry to evaluate the hydrodynamic performance of its multi-fin system. Our results demonstrate that in pufferfish steady swimming, caudal, dorsal and anal fin rays oscillate while performing significantly passive bending and twist deformations but show a noticeable out-of-phase feature, leading to neutralizing rotational forces and hence suppressing yaw motion, particularly at fast swimming. Numerical simulation suggests that the caudal median fin plays a key role in thrust generation while the dorsal and anal fins also provide a considerable contribution.
\end{abstract}

Keywords pufferfish, multi-fin, kinematics, deformation, hydrodynamics, CFD 


\section{Introduction}

The desire of investigation and exploitations on ocean resources propels the development of underwater vehicles in the past several decades. In nature, fish has superior swimming performance compared to artificial swimmers in many aspects, such as it can achieve fast speed, high efficiency and low noise, presenting sophisticated ventures in biomimetic designs of underwater vehicles. Mimicking the geometric and kinematics of fish is considered as a shortcut to absorb the preponderance of fish swimming into unmanned underwater vehicles (UUV).

Fish swimming modes are generally categorized into BCF (body and caudal fin) and MPF (median and paired fins) modes (Webb and Blake, 1985). BCF mode has good rapidity (e.g. tuna fish), while MPF mode provides good maneuverability. As a subtype of MPF, Tetradontiform swimmers such as pufferfish (Blake, 1983; Webb, 1984, 1994) oscillates pectoral, dorsal and anal fins independently. Those fins coordinate with body-caudal-fin undulation, forming specific gaits depending on the swimming speed. It is observed that pufferfish swim with pectoral, dorsal, anal and caudal fins at lower speed, but at medium-high speed, with the pectoral fins locked coherently attaching onto the body to reduce drag. As an extreme example of Tetradontiform swimmers, boxfish have a rigid body and utilize multi-fins for propulsion and maneuvering (Blake, 1977; Gordon et al., 2000; Walker, 2000; Hove et al., 2001).

Hydrodynamics in fish swimming have been studied through experimental, analytical and computational approaches. Although analytical models, mainly based on elongated-body theory (Fish and Lauder, 2006; Lighthill, 1990a, 1990b, 1990c, 1990d) that has been widely used, are effective means in studying BCF fish, the swimming with multiple flexible fins and plump body is intractable case for them. Thus, the hydrodynamics of Tetradontiform swimmers are primarily examined through experimental and computational approaches. Recently, hydrodynamics of BCF fish has been studied by experimental observation with PIV (Particle Image Velocimetry) technique, which has been applied to assess fin-based thrust enhancement for MPF swimming in the pufferfish (Wu, 2001; Breder, 1926; Blake and Chan, 2011). Computational fluid dynamic (CFD) modeling of hydrodynamics and free-swimming body dynamics that couples the Navier-Stokes (NS) equations to the equations of undulating body motion with pectoral fins has been also developed and employed in unveiling free-swimming hydrodynamics in fish (Liu et al., 1996, 1997,1999; Liu, 2017; Katumata et al. 2009; Li et al. 2011, 2012, 2016).

With respect to the flexible fins in swimming, both passive and active deformation-based control mechanisms have been explored till now for MPF mode (Webb, 2006; Bartol et al., 
2005). In the pufferfish swimming, fin and body movements serve in powered control mechanisms, while integumentary ornamentation (e.g., spines) and skin compliance properties are the possible mechanisms for unconscious control (Brainerd, 1994; Gordon et al., 1996; Arreola and Westneat, 1996). However, how fin flexibility influences the hydrodynamics and maneuverability in particular with multi-fin system in pufferfish swimming remains unclear yet.

In this study we aim at unveiling novel mechanisms associated with multi-fin kinematics and hydrodynamic performance in pufferfish steady swimming through an integrated study by combining measurement and digitizing of multi-fin kinematics and three-dimensional deformations and CFD modeling of steady cruising swimming. We first measured the kinematics and deformations of dorsal, anal, and caudal fins with two high-speed cameras by filming a free-swimming pufferfish at specific speeds in circulating water channel. We then digitized and performed a comprehensive analysis of the multi-fin kinematics and deformations in terms of active and passive fin deformations. We further built up a CFD model of the pufferfish with a realistic body and multi-fin geometry to evaluate the hydrodynamic performance of its multi-fin system. Finally we gave an extensive discussion on the effects of flexible fins on multi-fin kinematics and hydrodynamic.

\section{Material and methods}

\subsection{Experimental set-up}

\subsubsection{Pufferfish and circulating water channel}

Pufferfish (Teleostei: Diodon holocanthus) were purchased from a local aquarium and kept in a water tank for a week, which had a body length of $11.4 \pm 0.2 \mathrm{~cm}$ (averaged based on five measurements). Artificial seawater (density: $1.022 \pm 0.001 \mathrm{~kg} \cdot \mathrm{m}^{-3}$, temperature: $26 \pm 2^{\circ} \mathrm{C}$ ) was used in the water tank. The experiments were conducted in a circulating water channel (Fig. 1) in Jiangsu University of Science and Technology, China. The circulating water channel system was comprised of a water channel, converter pumps and a control cabinet. The working section of the circulating water channel was $50 \times 30 \times 40 \mathrm{~cm}(\mathrm{~L} \times \mathrm{W} \times \mathrm{H})$. In order to achieve a uniform incoming flow, a rectifying plate was placed in front of the water channel at upstream side. The same artificial seawater was also utilized in the water channel. The rotating speed of the converter pump was controlled by the control cabinet, which successfully achieved a flow velocity range over $0-45 \mathrm{~cm} / \mathrm{s}$.

\subsubsection{High-speed digital filming}

In order to record the three-dimensional motions of pufferfish, two high-speed digital cameras were set up in front of the working section of the water channel with a specific angle (Fig.1). The maximum resolution and frame rate of the cameras (Phantom ${ }^{\circledR}$ 
Miro ${ }^{\circledR}$ eX4) were $800 \times 600$ pixels and 1260 fps, respectively. The two cameras were controlled by a PC computer through data lines and switchboard, which sent commands to achieve synchronous recording of the two cameras with software (PCC 2.4). With a set-up of combining a resolution and a frame rate of $800 \times 600$ pixels and $100 \mathrm{fps}$, the cameras could complete a video recording up to 88 seconds. The software (PCC 2.4) provided a post-trigger function, which was instrumental in capturing the steady swimming of the pufferfish undergoing free-swimming in the circulating water channel at some given incoming speed.

\subsubsection{Calibration of filming}

For three-dimensional analysis, calibration was conducted to ensure the calculation of the precise locations of the cameras and to construct the three-dimensional coordinate system. The calibration requires at least 6 discrete points whereas extra points may further increase the accuracy of calibration. Here a calibration fixture was used, which was composed of two sheets with an angle between the two sheets of 120 degree. Each sheet contained 60 points with an interval (distance) between neighbor points of $2 \mathrm{~cm}$, and that from median line to the nearest points of calibration fixture of $1 \mathrm{~cm}$ (Fig. 2). Before recording the pufferfish swimming, the calibration fixture was placed in the working section of the circulating water channel. We confirmed that at least each camera could photograph 20 points on each sheet. The images containing the calibration points were then processed with three-dimensional motion analysis software (ProAnalyst, Xcitex) to reconstruct the three-dimensional Cartesian coordinates (Fig.2). The validity and accuracy of the calibration fixture was confirmed in advance by measuring the length of a ruler at three different locations, through contrast test and correction, a maximum error of $3.7 \%$ of the length was acceptably achieved.

\subsubsection{Experiment procedure}

In order to adapt the fish to the environment of the experiment, the selected pufferfish were trained to swim freely in the working section of the water tunnel several hours before filming. At the beginning of the experiments, the flow velocity was gradually increased from 0 up to $1.0 \mathrm{~L} / \mathrm{s}$, and then the two cameras were turned on synchronously to start filming and recording, which were terminated 5 seconds after when the pufferfish reached a steady state of swimming. All the filmed video was then transferred and saved to a computer. The same procedure was repeatedly carried out for eleven cases corresponding to different incoming flow speeds ranging over 1.0-3.0 L/s with an interval of $0.2 \mathrm{~L} / \mathrm{s}$.

\subsection{Kinematic analyses}

In order to determine body and multi-fin kinematics, ten stable tail beat cycles with sufficiently high resolution were chosen for the analysis of each case, which were defined as starting from and returning to the maximum (left or right) lateral excursion. The 3D coordinates and cycles selected from the videos were processed with software of ProAnalyst. 
As illustrated in Fig. 3 we set seven tracking points on the tip and two on the base of caudal, dorsal and anal fins, as well as one frame attached to each fin and one frame of reference attached to the body, respectively. Using reference frame attached to the fish can remove the periodical surge motion caused by the fluctuation of total force exerted on fish during analysis. Note that thirty tracking points in toto were set on the body, caudal, dorsal and anal fins on each time frame (Fig. 3). In all the experiments, the pectoral fins were observed to cling against body coherently with no any oscillating, and hence excluded from kinematic analysis.

To determine the amplitude of lateral excursion, the lateral translation of each tracking point was calculated as the distance diverged from the median plane of the body. To determine the angular amplitude of each fin ray, the angular displacement was calculated as the angles between fin rays and the median plane of the body (Fig. 3).

For the fin movement, the angular displacements of caudal, dorsal and anal fins in the body frame of reference were calculated by the following formulas:

$$
\begin{aligned}
& A_{c}=\arctan \left(\frac{y_{c}}{x_{c}}\right), \\
& A_{d / a}=\arctan \left(\frac{y_{d / a}}{z_{d / a}}\right),
\end{aligned}
$$

where $A_{c}$ denotes the angular displacement of a caudal fin ray, $x_{c}$ and $y_{c}$ their coordinates in the body frame of reference; $A_{d / a}$ expresses the angular displacement of a dorsal/anal fin ray, $y_{d / a}$ and $z_{d / a}$ their coordinates in the fin frame of reference. The body amplitude (Fig.3, B1, B3, B4) is represented by y-coordinates of the tracking points in the body frame of reference.

For a complete stroke cycle, all the displacements of the body, caudal, dorsal and anal fin rays were fitted with Fourier series as follows:

$$
\mathrm{A}=a_{0}+\sum_{i=1}^{n}\left(a_{i} \cos (n w t)+b_{i} \sin (n w t)\right), \quad(n=1,2)
$$

where $w$ denotes circular frequency, $t$ time, $a_{0}, a_{i}, b_{i}$ Fourier coefficients, respectively.

At speed of $1.0 \mathrm{~L} / \mathrm{s}$, the displacements of the caudal, dorsal and anal fin rays (Fig. 3, C4, D1, A1) were fitted with both first and second order Fourier series, which, as shown in Fig. 4, show a indistinctive difference between each other. The R-squares are further calculated and summarized in Table 1, demonstrating that both $1^{\text {st }}$ and $2^{\text {nd }}$ order Fourier series are of high accuracy in fitting the measured displacements. Therefore the 1st order Fourier series-based fitting was utilized for all the measured displacements.

The fin kinematics can then be expressed in a sinusoidal function, such that:

$$
\phi=A \sin (\omega t+\theta),
$$

where $A$ denotes amplitude, $\theta$ initial phase, $\omega$ circular frequency, and $t$ time. Since the fin deformation generally forms a three-dimensional surface, an interpolation based on cubic 
B-spline curve was adopted to reconstruct the deformation surfaces of caudal, dorsal and anal fins.

The opening width of each fin, as the indicator of fin deformation level being used for analysis of the variation in fin area, was defined as a distance in vertical plane between tracking points $\mathrm{C} 1$ and $\mathrm{C} 4, \mathrm{D} 1$ and $\mathrm{D} 7, \mathrm{~A} 1$ and $\mathrm{A} 7$, respectively, for caudal, dorsal and anal fins, and was calculated at all frames.

Phase difference between multiple fins is a key factor for producing thrust and side forces, which may play a vital role in terms of propulsion as well as maneuverability and stability in pufferfish swimming. Here the phase differences among caudal, dorsal and anal fins were determined by calculating the relative differences in maximum displacement time among the fins. Note that noticeable changes in beat frequency, amplitude, and phase difference in multiple fins may result in gait transitions.

\subsection{CFD modeling}

Follow Liu's method (Liu and Kawachi 1999; Nakata and Liu, 2012) that can determine 3D body-geometry using two 2D images of the object based on two pictures of side and top views, we defined the geometries of the pufferfish body (Teleostei: Diodon holocanthus) on the basis of side and top views (Fig.3), and the fins based on fin peduncle and outlines of dorsal, anal and caudal fins, respectively. As in the work by Gordon (Wiktorowicz et al., 2007) we defined the center of mass (CoM) of puffefish (Teleostei: Diodon holocanthus) at $34.4 \pm 0.3 \%$ BL posterior of the snout tip. The kinematic model for pufferfish swimming with consideration of deformations dorsal, anal and caudal fins was then defined to have a form of,

$$
\varphi(a, t)=A(a) \sin (\omega t+\theta(a)),
$$

where $A(a)$ represents amplitude, $\omega$ circular frequency, $t$ time, $\theta(a)$ initial phase, $\varphi(a, t)$ the center plane of pufferfish geometry model, and $a$ the angle position of fin rays, respectively (Fig. 3).

Given reference lengths of $L$ and $L_{c}$, a reference velocity $U$, and oscillating frequency $f$ the Reynolds number $\left(R_{e}\right)$ and Strouhal number $\left(S_{t}\right)$ are defined by

$$
R_{e}=\frac{U L}{v}, \quad S_{t}=\frac{f L_{c}}{U},
$$

where $v$ represents water kinematic viscosity of $0.92255 \times 10^{-6} \mathrm{~m}^{2} / \mathrm{s}, L$ is the body length of $11.4 \mathrm{~cm}, U$ is the forward speed, $f$ is the oscillating frequencies, and $L_{c}$ is the amplitude of caudal oscillation, respectively. The $R_{e}$ and $S_{t}$ are shown in Table 2.

Three-dimensional CFD models were built up based on geometric and kinematic data of the objective pufferfish, including a body, as well as caudal, dorsal and anal fins. Fig. 5 illustrates the layout of computational domain, which is taken sufficiently large to have a 
distance of 5 BL (Fig. 5, D1, D4) to side boundaries, 5 BL to the upstream boundary (Fig. 5, D2), and $10 \mathrm{BL}$ (Fig. 5, D3) to the downstream boundary, respectively. 2D unstructured triangular meshes were generated on the surfaces of pufferfish body and fins (Fig.5E) as well as on the six outside boundaries; and then $3 \mathrm{D}$ tetrahedron meshes were generated within the computational domain, with local meshes clustered to the surfaces of body and fins to ensure the boundary resolution adjacent to the solid surfaces (Fig.5F).

Commercial flow solver ANSYS FLUENT 16.0 was used in all the simulations. At upstream boundary, the swimming velocity is fixed while a pressure gradient is set to be zero. At downstream boundaries zero-gradient condition is imposed for both velocities and pressures. On the surfaces of body and fins, no-slip condition is employed for velocities. The time step was determined based on the Courant number (CFL). Mesh dependency was first investigated by introducing three different meshes of Mesh A, Mesh B and Mesh C with a minimum mesh sizes of $0.3 \mathrm{~mm}, 0.5 \mathrm{~mm}$ and $0.7 \mathrm{~mm}$ (Fig. $5 \mathrm{G}$ ), respectively, as well as two time steps of $0.001 \mathrm{~s}$ and $0.0005 \mathrm{~s}$, in terms of a comparison of resistance coefficient $\left(C_{d}\right)$ acting on the pufferfish model at $R e$ of $1.4087 \times 10^{4}$. Since the computed drag force shows less difference with a difference within $2 \%$ among the three meshes and the time steps (Fig. $5 \mathrm{G})$, we then utilized a combination of Mesh B and time step A for all the simulations. In this study, we simulated swimming at 1,2 and $3 \mathrm{BL} / \mathrm{s}$ identical to Reynolds numbers of $1.4087 \times 10^{4}, 2.8174 \times 10^{4}$ and $4.2252 \times 10^{4}$, respectively. According to previous experimental (Anderson et al. 2001) and computational studies (Liu and Kawachi, 1999) on undulatory swimming, the Reynolds number is below a critical transition level to turbulence, therefore laminar condition was used in the simulations.

\section{Results and discussion}

In order to investigate the multi-fin kinematics and their relationship with hydrodynamic performance in pufferfish swimming, we first analyzed the multi-fin kinematics by digitizing the fin kinematics and deformations in a three-dimensional manner, mainly in terms of passive deformations in caudal, dorsal and anal fins as well as phase differences among them. We then constructed a realistic multi-fin kinematic model for CFD modeling of the pufferfish with realistic body and multi-fin geometries to evaluate the hydrodynamic performance of its multi-fin system.

\section{1 Passive deformation of caudal, dorsal and anal fins}

Caudal fins play a key role in thrust generation and its flexibility may directly dominate the propulsion performance in pufferfish swimming. The outermost rays of caudal fins as illustrated in Fig. 3, C1 and C7 fin rays are driven by caudal peduncle, which in general show 
passive deformations throughout the entire caudal fin. It was observed that in steady swimming, the upper and lower halves of the caudal fin deformed symmetrically. Due to the disparity in flexibility of fin rays, outside fin rays show smaller amplitude rather than inner fin rays (Fig. 6). The amplitudes of all rays increased with increasing swimming velocity at low speed, but turned to decrease when the swimming speed reached sufficiently high. As shown in Fig.3, an opening length between points of $\mathrm{C} 1$ and $\mathrm{C} 7$ represents a change in windward area of caudal. $L_{\mathrm{C} 1}$ and $L_{\mathrm{C} 7}$ stood for the length of $\mathrm{C} 1$ and $\mathrm{C} 7$ rays, respectively. In one oscillating cycle, the opening length displayed periodic variation (Fig.9), and the period was half of oscillating cycle. To illustrate the change of opening length, change rate of length $d$ was used to represent the change with expression as follows:

$$
d=\frac{\left|L_{d}-L_{a}\right|}{L_{a}}
$$

where, $d$ denotes change rate of length, $L_{d}$ the maximum or minimum length in one oscillating cycle, and $L_{a}$ the average length, respectively.

From Table 3, the average opening length of one oscillating cycle changed little as the forward velocity varied from 1 to $3 \mathrm{BL} / \mathrm{s}$, and the maximum length deviation was about $5 \%$. Likewise, the lengths of $\mathrm{C} 1$ and $\mathrm{C} 7$ rays also opening length displayed periodic variation, and had slight change in one oscillating cycle (Table 4). This indicated a small change in the windward area at all velocities, so we can assume that the change in windward area of caudal fin at all velocities could be neglected.

As the leading-edge of fin rays, D1 and A1 fin rays play an active role in driving or oscillating dorsal and anal fins (Fig.3 and Fig.8). The phases of D1 and A1 rays were ahead of D7 and A7 rays which were trailing-edges. The amplitude of trailing-edge rays for dorsal and anal fins was smaller than those of the leading edges, and decreased with the increasing of swimming velocities and frequency. In steady swimming, it was observed that the motions of anal and dorsal trailing rays are in-phase and the phase difference between leading and trailing ray remains almost constant. We further show the change of ray length (D1, D7, A1 and A7) in one oscillating cycle (Fig.8 and Table 5), obviously, the length of rays also displayed periodic variation, and the change was small, which were less than $4 \%$ at all velocities.

Based on the above results and analysis, snap sequences of the motions and deformations of the caudal, dorsal and anal fins are illustrated in Fig. 9 in one complete beat with the methods described in previous section.

\subsection{Phase difference in multiple fins}

Specific phase difference in multi-fin system is often observed and important in pufferfish swimming in terms of propulsive performance and stability. Oscillation of one 
single fin can produce a thrust force but inevitably caused lateral recoil forces as well, which leads to yawing of fish in swimming, attenuate their oscillating amplitudes and hence lower the thrust generation. Yawing may also increase the fish frontal area and destabilize the streamlines on fish body, resulting in increasing the form drag (pressure drag). It was observed in our experiments that pufferfish seem to utilize a specific phase difference among caudal, dorsal, anal fins as well as caudal peduncle (Fig. 10A) to eliminate the side forces and hence the yaw motions: the caudal fin is in phase with caudal peduncle, while dorsal and anal fins are also in phase with each other; but the caudal fin is out of phase with dorsal and anal fins. Furthermore, interestingly a specific phase difference between caudal and dorsal is seen at all velocities with almost no variation.

As an indicator of the yawing, we here introduced a heading angle (angle between the heading direction and the forward direction), which is observed to vary with different swimming velocity. As shown in Fig. 10B, the heading angle presents a significant decrement trend when the swimming velocity is greater than $2.4 \mathrm{BL} / \mathrm{s}$.

\subsection{Oscillating frequency and amplitude in multiple fins}

In steady swimming, as plotted in Fig.10C, caudal, dorsal and anal fins share the same oscillating frequency, which appears to be proportional to the swimming velocity. Amplitudes of caudal, dorsal and anal fins, however, vary with swimming velocity, showing a peak around a velocity of $2.0 \mathrm{BL} / \mathrm{s}$ in dorsal and anal fins but some peak around a velocity of 2.2 BL/s in caudal fin (Fig.10D). It suggests that pufferfish employ a strategy of swimming faster by increasing oscillating frequency of multiple fins instead of enlarging the amplitude.

\subsection{Hydrodynamics of multiple fins}

With a realistic multi-fin kinematic model and a CFD model of the pufferfish with realistic body and multi-fin geometries (Fig. 11), we carried out the simulations of steady swimming at three velocities of 1.0, 2.0,3.0 BL/s. With consideration of a trimmed steady swimming, it is important to calculate and evaluate the three forces of $F x, F y$, and $F z$ in $\mathrm{x}, \mathrm{y}$, z-directions, as well as three moments (torques) around three axes. The center of buoyancy was determined by the geometric model of pufferfish, located at $36 \%$ BL (Fig.11). For pufferfish, the precise location of the center of mass was difficult to determine. Based on previous study (Wiktorowicz et al., 2007), in order to explore the effects of position of mass center on moment balance, we respectively set the center of mass at 0.00(CM1), 0.02(CM2), 0.04 (CM3) BL under the center of buoyancy in simulations (Fig.11). In pufferfish self-propulsion swimming, body produced the drag force, including pressure and friction resistance. In simulations, by integrating pressure and friction resistance over body, body drag force can be obtained. In the same way, we can also get the thrust force of caudal, dorsal and 
anal fins. Then the drag and moment coefficients can be determined by the expressions as follows:

$$
C_{d}=\frac{D}{\frac{1}{2} \rho U^{2} S}, C_{m}=\frac{M}{\frac{1}{2} \rho U^{2} S L},
$$

where, $C_{d}$ is drag coefficient; $C_{m}$ is moment coefficient; $\rho$ is density of water; $U$ is velocity; $S$ is reference cross section area of pufferfish; $L$ is body length.

As summarized in Table 6, it is seen that the fins-based thrust coefficients in $\mathrm{x}$-direction (horizontal, forward-backward direction) can approximately balance the body-based drag coefficients at all the three swimming velocities, with mild difference rates between thrust and drag of $-3.8 \%,-2.59 \%$ and $11.05 \%$, respectively. Here, it is interesting to note that the dorsal and anal fin-based thrust is much greater than that by the caudal fin. In y-direction (vertical direction), the resultant force coefficients are slightly greater than zero in all swimming velocity scenarios with a net lift force being less than approximately $0.85 \%$ of buoyancy. For a real pufferfish, the swim bladder may adjust its own weight to keep its balance. In z-direction (horizontal, lateral direction), both fins-based and body-based forces are minimal compared with those in $\mathrm{x}$-and $\mathrm{y}$-direction.

Since the center of mass is lower than buoyancy, when the fish body declines, the buoyancy could generate a restore moment to maintain the body balance. For instance, with swimming velocity of $1.0 \mathrm{BL} / \mathrm{s}$, as summarized in Table $7, \overline{M_{z}}$ is generated as a clockwise moment against an anticlockwise moment owing to buoyancy, which in toto maintain the pitching balance. The restore mechanism may work at all swimming velocities and around $\mathrm{x}$ $y$ - and z-axis, the resultant moments are calculated to be less than $5 \%$, indicating that the appropriate distance, a gap between the centers of mass and buoyancy in pufferfish is very important in their swimming stability and probably maneuverability as well.

Flow fields around pufferfish in steady swimming were further visualized in terms of velocity vectors, pressure distributions on body-fin surfaces, and wake topologies. As depicted in Fig. 12, one vertical cross-section and three horizontal cross-sections were chosen. From Fig.13, the maximum flow velocities in vertical cross-section were generated near caudal fin. In horizontal cross-sections (Fig. 13), flow field of caudal fin had been affected to some extent by dorsal fin oscillating, but not strongly. However, anal the oscillating of fin lead to a great influence on flow field of caudal fin. From vertical cross-section and three horizontal cross-sections, the oscillation of the fins synchronously and periodically produced vortices that shed symmetrically in to vortex street, which is a major factor in thrust generation.

Associated with the pressure distributions shown in Fig. 13, the maximum pressure was observed at the trailing edge of caudal fin. The highest normal pressure passed from leading 
edge to trailing edge on median fins. The complex time-varying uneven pressure distribution on fin surfaces resulted in a complex spatial deformation of fins. Oscillating of caudal, dorsal, and anal fins caused periodical change in body orientation and resulted in extra drag on body.

The vortex structures including the trailing vortices and wake topologies were further visualized in terms of Q criteria as depicted in Fig. 14. The vortex sheet in the wake was characterized by the hairpin vortex structure with three rows of vortex chains, induced by dorsal fin (upper chain), anal and caudal fins (lower chain) and tail fin (lateral chain), respectively. Obviously, the wake topology generated by the interplay among three fins in MPF swimming mode appears to be spatially more complicated compared with that observed in BCF swimming mode (Fish and Lauder, 2006).

\section{Conclusions}

The kinematics and hydrodynamics concerning the multiple fins of Pufferfish are studied. Our results demonstrated that pufferfish swim with caudal, dorsal and anal fins, while pectoral fins cling to the body in order to reduce resistance. In steady swimming, caudal, dorsal and anal fin rays perform small bending deformation during oscillation. All fins are driven by their respective leading fin ray, which leads to the pronounced passive movements. Observations from our experiment showed that dorsal fin oscillates in phase with anal fins, but out of phase with caudal fin. Such a phase difference phenomena is considered as a means to reduce the yawing, especially at high swimming speed, which is consistent with our experimental observations. Numerical results of the simulation demonstrated the rationality of CFD model of pufferfish, i.e. under steady swimming, median fins provide the majority of thrust, while dorsal and anal fins can contribute thrust as large as the caudal fin. These findings will help to deepen the understanding of the novel mechanisms of pufferfish swimming, and thus provide useful information to the bioinspired swimming robot design.

\section{Acknowledgements}

HL is partly supported by the Grant-in-Aid for Scientific Research on Innovative Areas of No. 24120007, JSPS. GL is partly supported by JSPS KAKENHI Grant Number JP17K17641 .

\section{Reference}

Anderson E.J., McGillis W.R., Grosenbaugh M.A. 2001 The boundary layer of swimming fish. J. Exp. Biol. 204, 81 -102.

Arreola, V.I., Westneat, M.W., 1996. Mechanics of propulsion by multiple fins: kinematics of aquatic locomotion in the burrfish (Chilomycterus schoepfi). Proc. R. Soc. Lond. Ser. B, $263,1689-1696$ 
Bartol, I.K, Gharib, M., Webb, P.W., Weihs, D., Gordon, M.S., 2005. Body-induced vortical flows: a common mechanism for self-corrective trimming control in boxfishes. J Exp. Biol. 208,327-344.

Blake, R. W., 1977. On ostraciiform locomotion. J. Mar. Biol. Ass. U.K., 57, 1047-1055.

Blake, R. W., 1983. Fish Locomotion. New York: Cambridge University Press.

Blake, R. W., Chan, K. H. S., 2011. Biomechanics of swimming in the pufferfish Diodon holocanthus: propulsive momentum enhancement is an adaptation for thrust production in an undulatory median and paired-fin swimmer. Journal of Fish Biology. 79, 1774-1794.

Brainerd, E.L., 1994. Pufferfish inflation: functional morphology of postcranial structures in Diodon holocanthus (Tetraodontiformes). J Morphol. 220(3), 243-261.

Breder, C. M., 1926. The locomotion of fishes. Zoologica. 4, 159-297.

Fish, F.E., Lauder, G.V., 2006. Passive and active flow control by swimming fishes and mammals. Annu Rev Fluid Mech. 38,193-224.

Gordon, M.S, Plaut, I., Kim, D., 1996. How puffers (Teleostei:Tetraodontidae) swim. J Fish. Biol. 49,319-328.

Gordon, M.S., Hove J.R., Webb P.W., Weihs, D., 2000. Boxfishes as unusually well-controlled autonomous underwater vehicles. Physiol. Biochem. Zool, 73(6), 663671.

Hove, J. R., O’Bryan, L. M., Gordon, M. S., Webb, P. W., Weihs, D., 2001. Boxfishes (Teleostei: Ostraciidae) as a model system for fishes swimming with many fins: kinematics. J. Exp. Biol. 204, 1459-1471.

Katumata, Y., Müller, U.K. and Liu, H., 2009. Computation of self-propelled swimming in larva fishes. J. Biomech. Eng. 4, 54-66.

Li, G., Liu, H., Müller, U.K., Leeuwen, J.L., 2011. Swimming hydrodynamics and maneuverability in C-start of zebrafish larvae: an integrated computational study. ASME-JSME-KSME 2011 Joint Fluids Engineering Conference. 1, 2059-2066.

Li, G., Müller, U.K., Leeuwen, J. L., Liu, H., 2012. Body dynamics and hydrodynamics of swimming fish larvae: a computational study. J. Exp. Biol. 215:4015-4033.

Li, G., Müller, U.K., Leeuwen, J. L., Liu, H., 2016. Fish larvae exploit edge vortices along their dorsal and ventral fin folds to propel themselves. J. R. Soc. Interface 13, 20160068.

Lighthill, J., Blake, R. W., 1990a. Biofluiddynamics of balistiform and gymnotiform locomotion. Part 1. Biological background, and analysis of elongated-body theory. Journal of Fluid Mechanics. 212, 183-207.

Lighthill, J., 1990b. Biofluiddynamics of balistiform and gymnotiform locomotion. Part 2. The pressure distribution arising in two-dimensional irrotational flow from a general symmetrical motion of a flexible flat plate normal to itself. Journal of Fluid Mechanics. 
213, 1-10.

Lighthill, J., 1990c. Biofluiddynamics of balistiform and gymnotiform locomotion. Part 3. Momentum enhancement in the presence of a body of elliptic cross-section. Journal of Fluid Mechanics. 213, 11-20.

Lighthill, J., 1990d. Biofluiddynamics of balistiform and gymnotiform locomotion. Part 4. Short-wavelength limitations on momentum enhancement. Journal of Fluid Mechanics, 213, 21-28.

Liu, H., Wassersug, R., Kawachi, K., 1996. A computational fluid dynamics study of tadpole swimming. J. Exp. Biol. 199, 1245-1260.

Liu, H., Wassersug, R., Kawachi, K., 1997. The three-dimensional hydrodynamics of tadpole locomotion. J. Exp. Biol. 200, 2807-2819.

Liu, H., Kawachi, K. 1999. A numerical study of undulatory swimming. J. Comput. Phys. 155, 223-247.

Liu, H., Kolomenskiy, D., Nakata, K., Li, G., 2017. Unsteady bio-fluid dynamics in flying and swimming. Acta Mechanica Sinica. 33, 663-684.

Nakata, T., Liu, H., 2012. A fluid-structure interaction model of insect flight with flexible wings. Journal of Computational Physics. 231, 1822-1847.

Walker, J. A. Does a rigid body limit maneuverability? J. Exp. Biol. 2000, 203, 3391-3396.

Webb, P. W., 1984. Body form, locomotion and foraging in aquatic vertebrates. American Zoologist 24, 107-120.

Webb, P. W. and Blake, R. W., 1985. Swimming in functional vertebrate morphology (ed. M. Hildebrand, D. M. Bramble, K. Liem and D. B. Wake). Cambridge, MA: Harvard University Press, 110-128.

Webb, P. W., 1994. The biology of fish swimming. In Mechanics and Physiology of Animal Swimming (Maddock, L., Bone, Q. \& Rayner, J. M. V., eds). Cambridge, U.K.: Cambridge University Press, 45-62.

Webb, P.W., 2016. Stability and maneuverability, In: Shadwick RE, Lauder GV (eds) Fish biomechanics. Elsevier and Academic, Amsterdam. 281-332.

Wiktorowicz, A.M, Lauritzen, D.V., Gordon, M.S., 2007. Powered control mechanisms contributing to dynamically stablswimming in porcupine puffers (Teleostei: Diodon holocanthus). Exp. Fluids 43,725-735.

$\mathrm{Wu}, \mathrm{Y} ., 2$ 2001. Mathematical bio-uiddynamics and mechanophysiology of fish locomotion. Math. Meth. Appl. Sci. 24, 1541-1564. 


\section{List of tables}

Table 1 Fitted R-squares of caudal, dorsal and anal fin rays at points of C4, D1, A1

\begin{tabular}{cccc}
\hline & $\mathrm{C} 4$ & $\mathrm{D} 1$ & $\mathrm{~A} 1$ \\
\hline First order & 0.9777 & 0.9816 & 0.9951 \\
Second order & 0.9871 & 0.9833 & 0.9957 \\
\hline
\end{tabular}

Table 2 Reynolds number $\left(R_{e}\right)$ and Strouhal number $\left(S_{t}\right)$

\begin{tabular}{cccc}
\hline $\begin{array}{c}\text { Forward speed } \\
(\mathrm{BL} / \mathrm{s})\end{array}$ & 1.0 & 2.0 & \\
\hline$f(\mathrm{~Hz})$ & 3.4 & 5.2 & 6.0 \\
\hline$L_{c}(\mathrm{~cm})$ & 0.91 & 0.94 & 0.92 \\
\hline$R_{e}$ & $1.4087 \times 10^{4}$ & $2.8174 \times 10^{4}$ & $4.2252 \times 10^{4}$ \\
$S_{t}$ & 0.272 & 0.215 & 0.173 \\
\hline
\end{tabular}

Table 3 Change rate of opening length at different swimming speeds

\begin{tabular}{cccccccccccc}
\hline & \multicolumn{11}{c}{ Swimming speed (BL/s) } \\
& 1.0 & 1.2 & 1.4 & 1.6 & 1.8 & 2.0 & 2.2 & 2.4 & 2.6 & 2.8 & 3.0 \\
\hline $\begin{array}{c}\text { Change rate of } \\
\text { opening length } \\
(\%)\end{array}$ & 3.0 & 3.5 & 4.2 & 4.3 & 4.5 & 5.1 & 4.3 & 4.9 & 5.2 & 4.7 & 5.2 \\
\hline
\end{tabular}

Table 4 Change rate at different swimming speeds

\begin{tabular}{|c|c|c|c|c|c|c|c|c|c|c|c|c|}
\hline \multicolumn{13}{|c|}{ Swimming speed $(\mathrm{BL} / \mathrm{s})$} \\
\hline & & 1.0 & 1.2 & 1.4 & 1.6 & 1.8 & 2.0 & 2.2 & 2.4 & 2.6 & 2.8 & 3.0 \\
\hline $\begin{array}{c}\text { Change rate } \\
\text { of ray }\end{array}$ & C1 ray & 3.4 & 3.7 & 3.8 & 3.8 & 3.5 & 4.4 & 3.6 & 3.8 & 3.9 & 3.9 & 4.1 \\
\hline length (\%) & C7 ray & 3.7 & 3.7 & 3.8 & 4.0 & 3.5 & 4.5 & 3.7 & 4.1 & 4.1 & 4.0 & 4.2 \\
\hline
\end{tabular}


Table 5 Changing rate of rays

\begin{tabular}{|c|c|c|c|c|c|c|c|c|c|c|c|c|}
\hline & \multicolumn{12}{|c|}{ Swimming speed (BL/s) } \\
\hline & & 1.0 & 1.2 & 1.4 & 1.6 & 1.8 & 2.0 & 2.2 & 2.4 & 2.6 & 2.8 & 3.0 \\
\hline \multirow{4}{*}{$\begin{array}{l}\text { Change } \\
\text { rate of ray } \\
\text { length } \\
(\%)\end{array}$} & D1 ray & 3.0 & 3.2 & 3.1 & 3.4 & 3.6 & 3.7 & 3.8 & 3.8 & 3.5 & 3.3 & 3.4 \\
\hline & D7 ray & 3.3 & 3.5 & 3.4 & 3.7 & 3.6 & 3.9 & 3.9 & 3.8 & 3.7 & 3.4 & 3.4 \\
\hline & A1 ray & 3.1 & 3.3 & 3.4 & 3.6 & 3.5 & 3.7 & 3.9 & 4.0 & 3.9 & 3.6 & 3.3 \\
\hline & A7 ray & 2.9 & 3.1 & 3.1 & 3.2 & 3.4 & 3.6 & 3.7 & 3.6 & 3.7 & 3.5 & 3.1 \\
\hline
\end{tabular}

Table 6 Mean force of body, caudal, dorsal and anal fins in one cycle

\begin{tabular}{|c|c|c|c|c|c|c|}
\hline \multirow{2}{*}{\multicolumn{2}{|c|}{$\begin{array}{l}\text { Speed } \\
(\mathrm{L} / \mathrm{s})\end{array}$}} & \multicolumn{4}{|c|}{ Force coefficient } & \multirow{2}{*}{$\begin{array}{l}\text { Resultant } \\
\text { force } \\
\text { coefficient }\end{array}$} \\
\hline & & caudal & dorsal & anal & body & \\
\hline \multirow{3}{*}{1.0} & $\overline{F_{x}}$ & -0.091 & -0.201 & -0.145 & 0.421 & -0.016 \\
\hline & $\overline{F_{y}}$ & 0.006 & 0.018 & 0.019 & 0.024 & 0.067 \\
\hline & $\overline{F_{z}}$ & 0.001 & -0.004 & -0.002 & -0.001 & -0.006 \\
\hline \multirow{3}{*}{2.0} & $\overline{F_{x}}$ & -0.076 & -0.099 & -0.063 & 0.232 & -0.006 \\
\hline & $\overline{F_{y}}$ & 0.004 & 0.064 & -0.022 & -0.005 & 0.041 \\
\hline & $\overline{F_{z}}$ & -0.003 & 0.006 & 0.003 & 0.007 & 0.013 \\
\hline \multirow{3}{*}{3.0} & $\overline{F_{x}}$ & -0.043 & -0.054 & -0.056 & 0.172 & 0.019 \\
\hline & $\overline{F_{y}}$ & -0.001 & 0.036 & -0.019 & -0.011 & 0.005 \\
\hline & $\overline{F_{z}}$ & -0.004 & 0.007 & 0.002 & 0.006 & 0.011 \\
\hline
\end{tabular}

Table 7 Mean resultant moments with different centers of mass in one cycle

\begin{tabular}{|c|c|c|c|c|c|c|c|c|c|}
\hline \multirow{3}{*}{$\begin{array}{l}\text { Resultant } \\
\text { moment } \\
\text { coefficient }\end{array}$} & \multicolumn{9}{|c|}{ Swimming speed (BL/s) } \\
\hline & & 1.0 & & & 2.0 & & & 3.0 & \\
\hline & $\begin{array}{l}\mathrm{dH}= \\
0 \mathrm{BL}\end{array}$ & $\begin{array}{c}\mathrm{dH}= \\
0.02 \mathrm{BL}\end{array}$ & $\begin{array}{c}\mathrm{dH}= \\
0.04 \mathrm{BL}\end{array}$ & $\begin{array}{l}\mathrm{dH}= \\
0 \mathrm{BL}\end{array}$ & $\begin{array}{c}\mathrm{dH}= \\
0.02 \mathrm{BL}\end{array}$ & $\begin{array}{c}\mathrm{dH}= \\
0.04 \mathrm{BL}\end{array}$ & $\begin{array}{l}\mathrm{dH}= \\
0 \mathrm{BL}\end{array}$ & $\begin{array}{c}\mathrm{dH}= \\
0.02 \mathrm{BL}\end{array}$ & $\begin{array}{c}\mathrm{dH}= \\
0.04 \mathrm{BL}\end{array}$ \\
\hline $\begin{array}{c}\overline{M_{x}} \\
\left(\times 10^{-2}\right)\end{array}$ & 0.31 & 0.25 & 0.17 & 0.32 & 0.26 & 0.21 & 0.37 & 0.31 & 0.27 \\
\hline $\begin{array}{c}\overline{M_{y}} \\
\left(\times 10^{-2}\right)\end{array}$ & 0.53 & 0.44 & 0.40 & -0.24 & -0.18 & -0.13 & -0.29 & -0.24 & -0.18 \\
\hline $\begin{array}{c}\overline{M_{z}} \\
\left(\times 10^{-1}\right)\end{array}$ & 0.1153 & 0.1101 & 0.0921 & 0.3256 & 0.3176 & 0.3047 & 0.2608 & 0.2316 & 0.2106 \\
\hline
\end{tabular}




\section{List of figures}

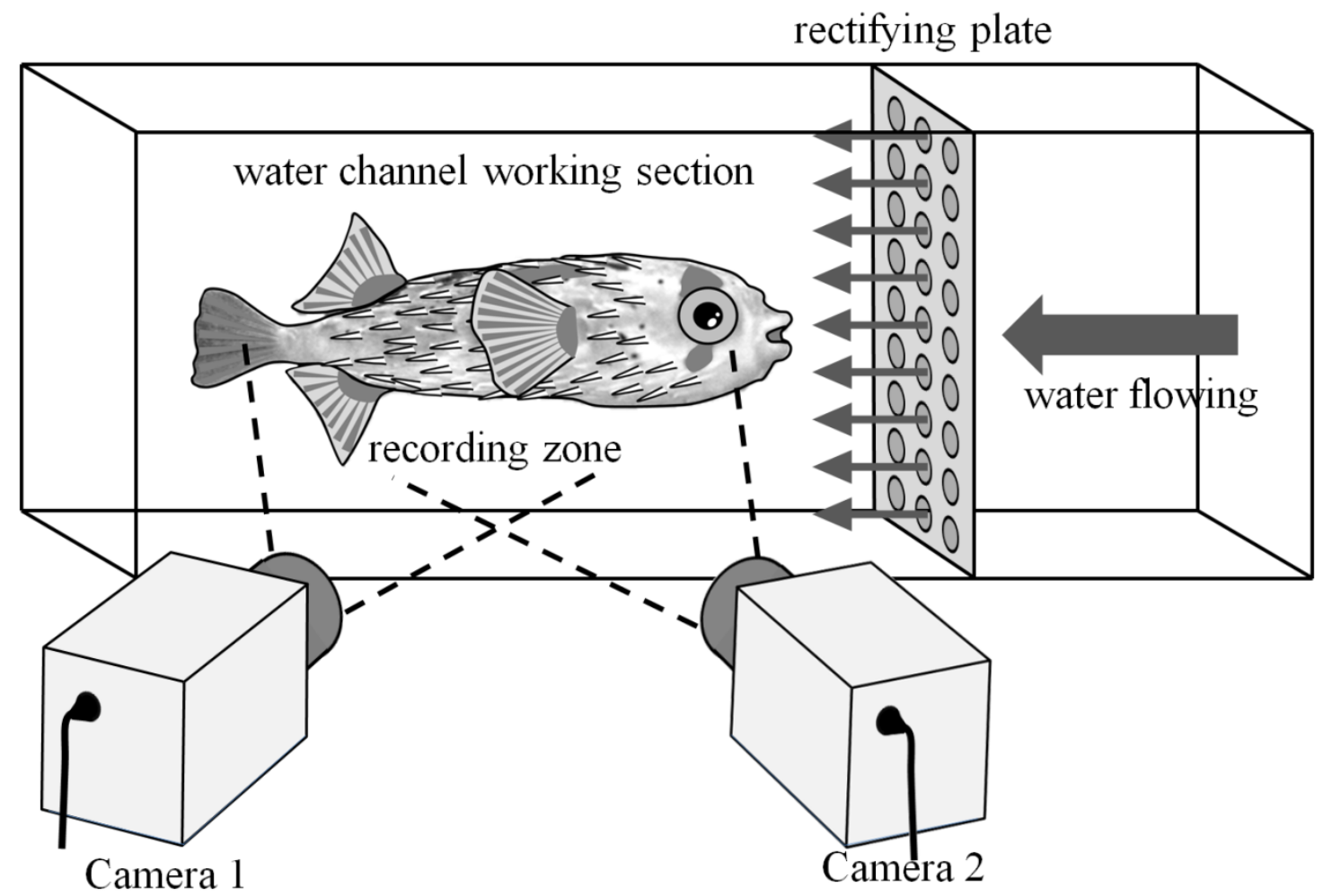

Fig.1 Sketch of experimental set-up involving the objective pufferfish, a circulating water channel, and a high-speed digital filming system. 


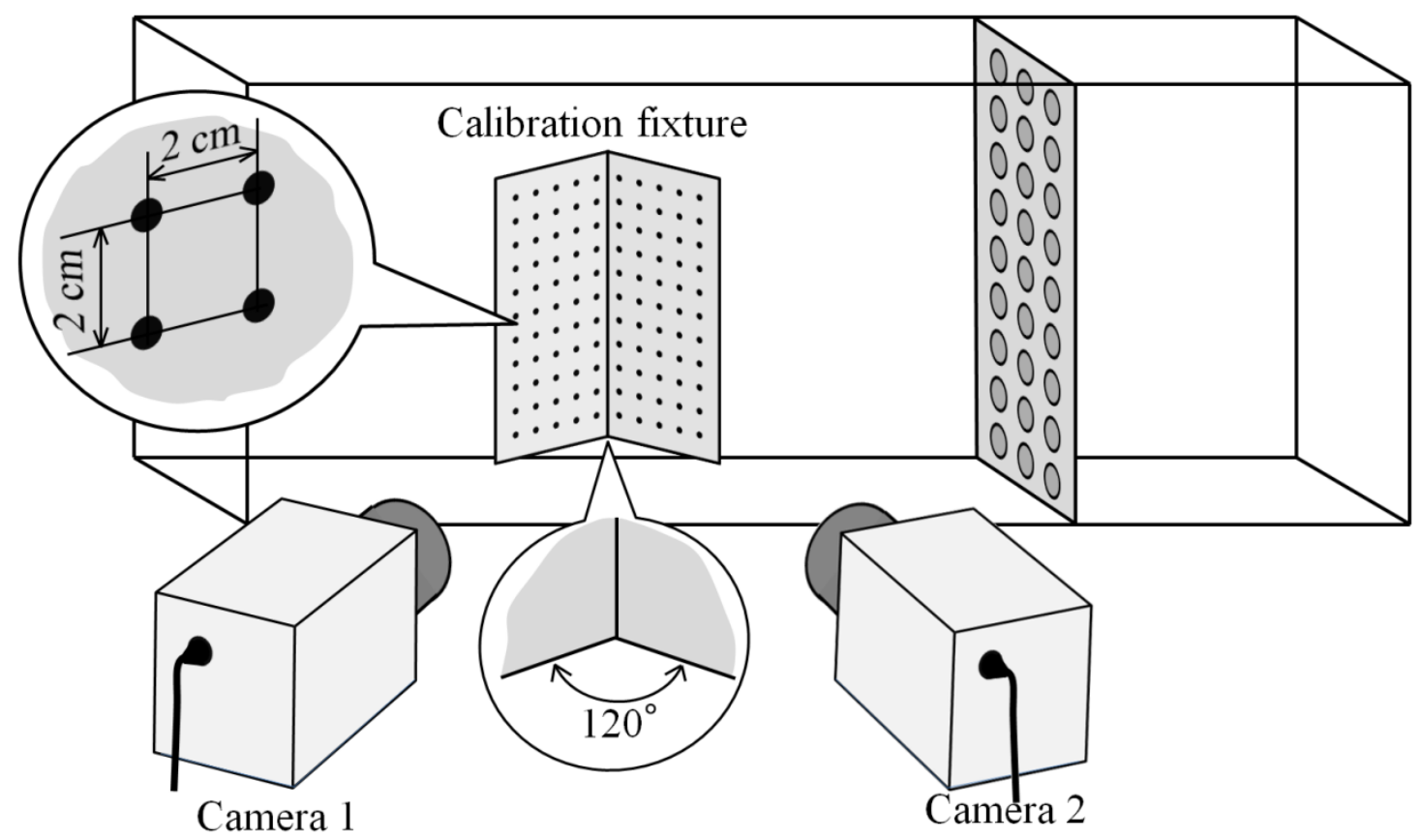

Fig. 2 Sketch of calibration fixture composed of two sheets with an angle of 120 degree and 60 black points with a distance of $2 \mathrm{~cm}$ apart on each sheet. 


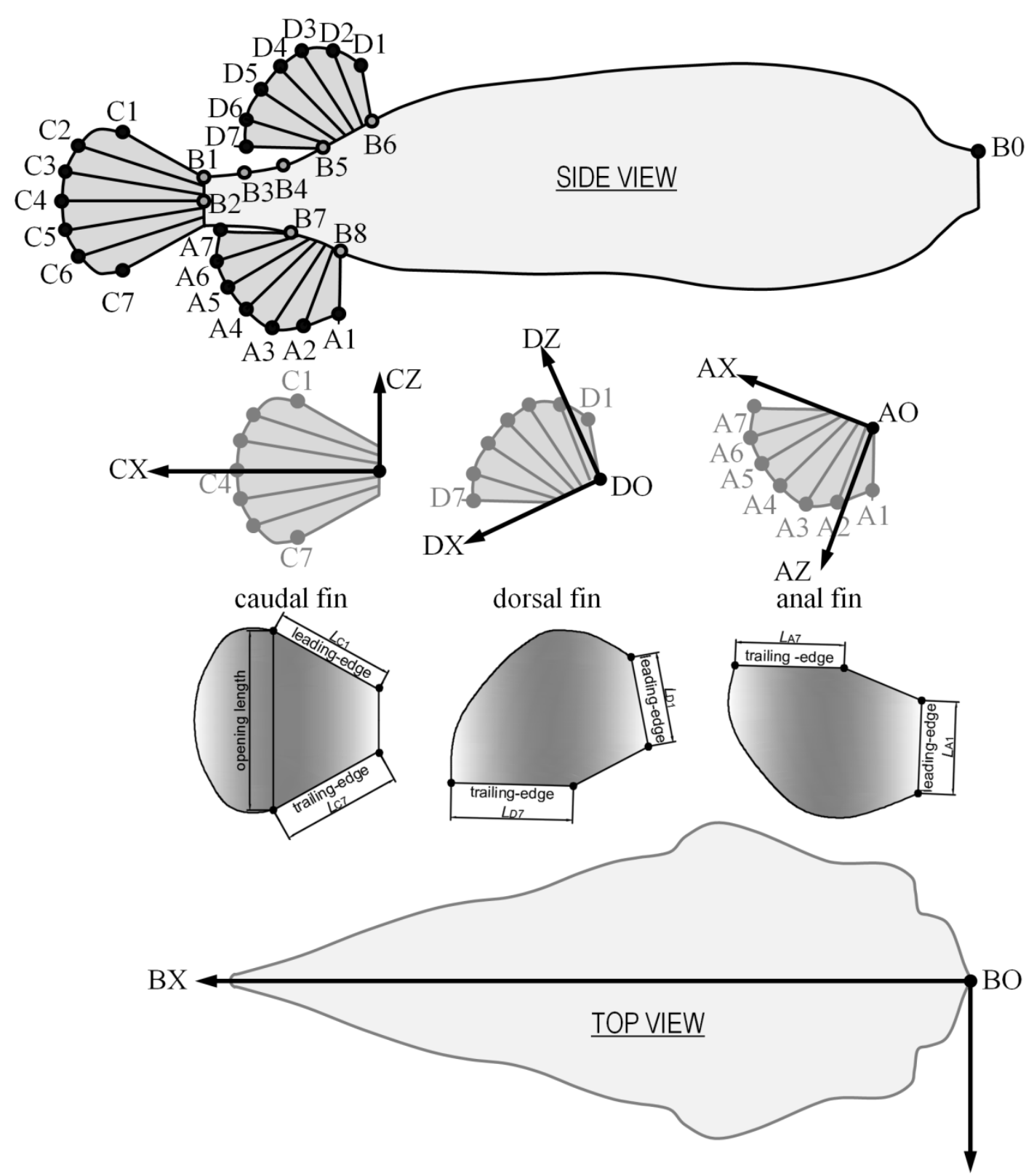

BY

Fig. 3 (Upper) Tracking points setting; (Middle) Attached frames and deformation parameters of caudal, dorsal and anal fins, respectively; (Lower) frame of reference attached to body. 

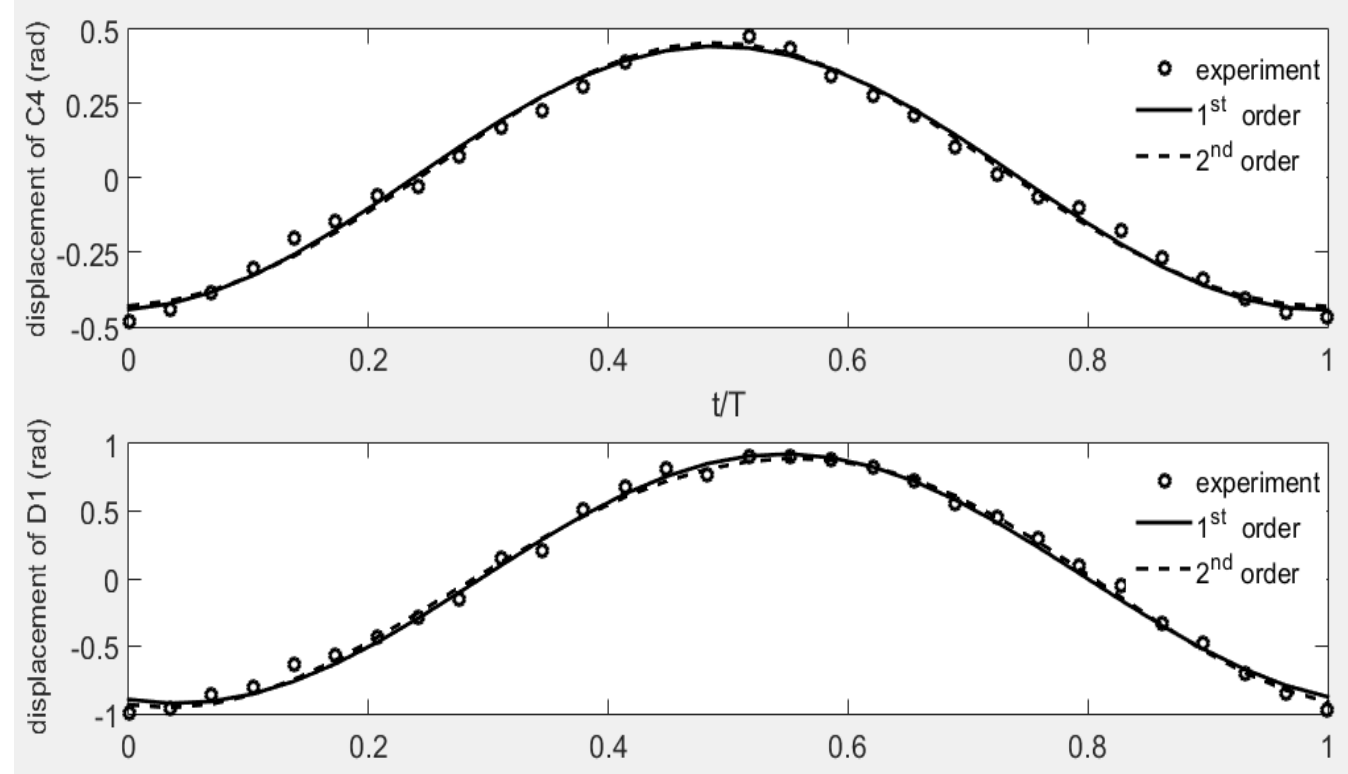

$\mathrm{t} / \mathrm{T}$

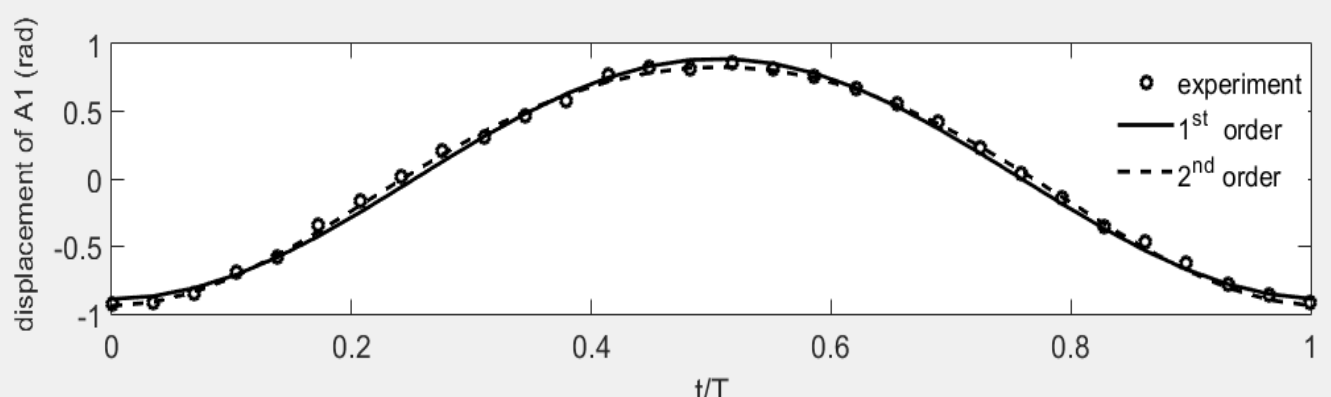

Fig. 4 Comparison between the measured and fitted displacements of caudal, dorsal and anal fin rays at points of $\mathrm{C} 4, \mathrm{D} 1, \mathrm{~A} 1$. 

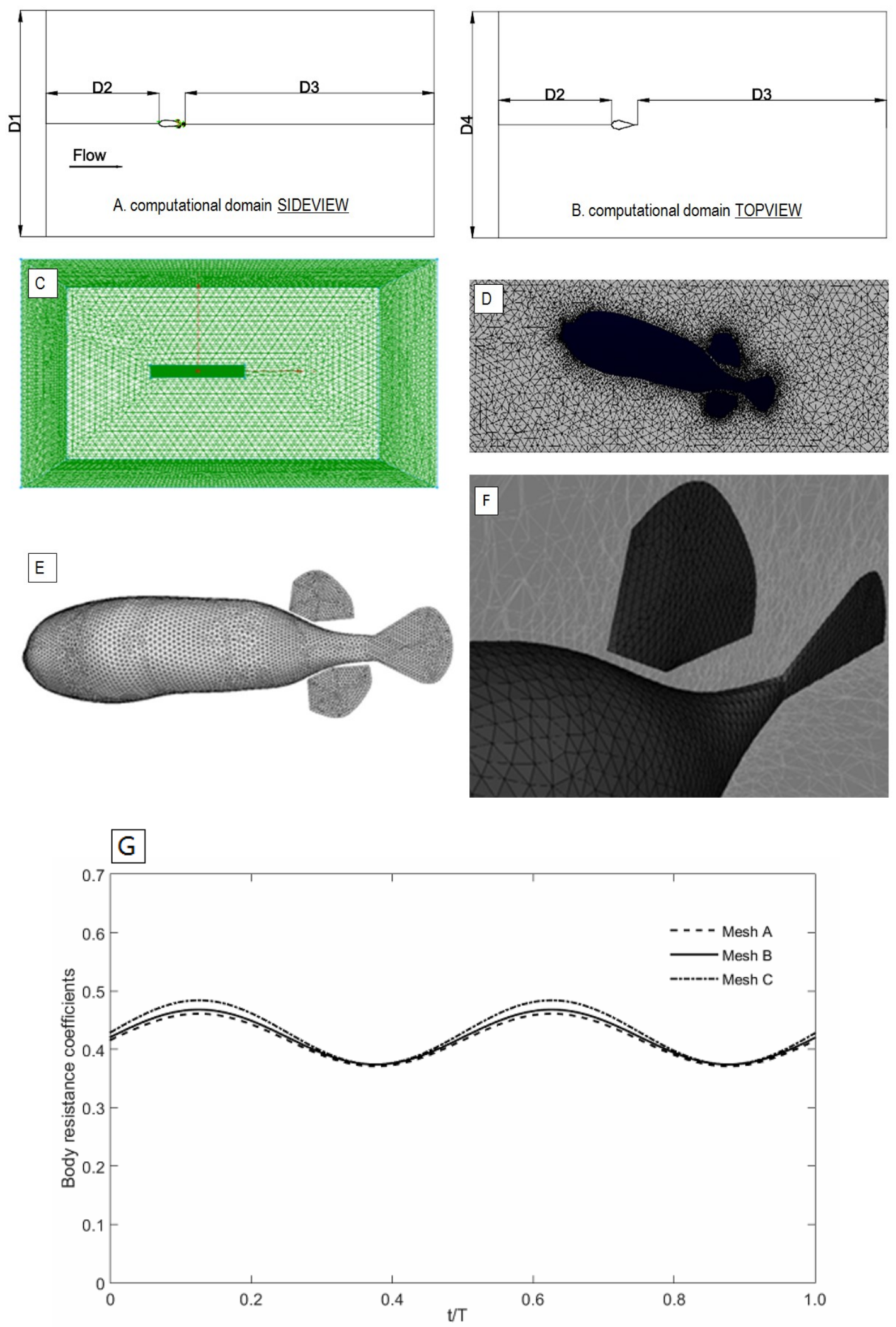

Fig. 5 Layout of computational domain: (A) Sideview; (B)Topview (C) meshes of computational domain (D) close-up view of meshes close to body-fin model; (E) Body surface meshes of pufferfish, and (F) Local mesh clustering adjacent to body and fin surfaces (G) Comparison of time-varying drag coefficients among three grid systems. 

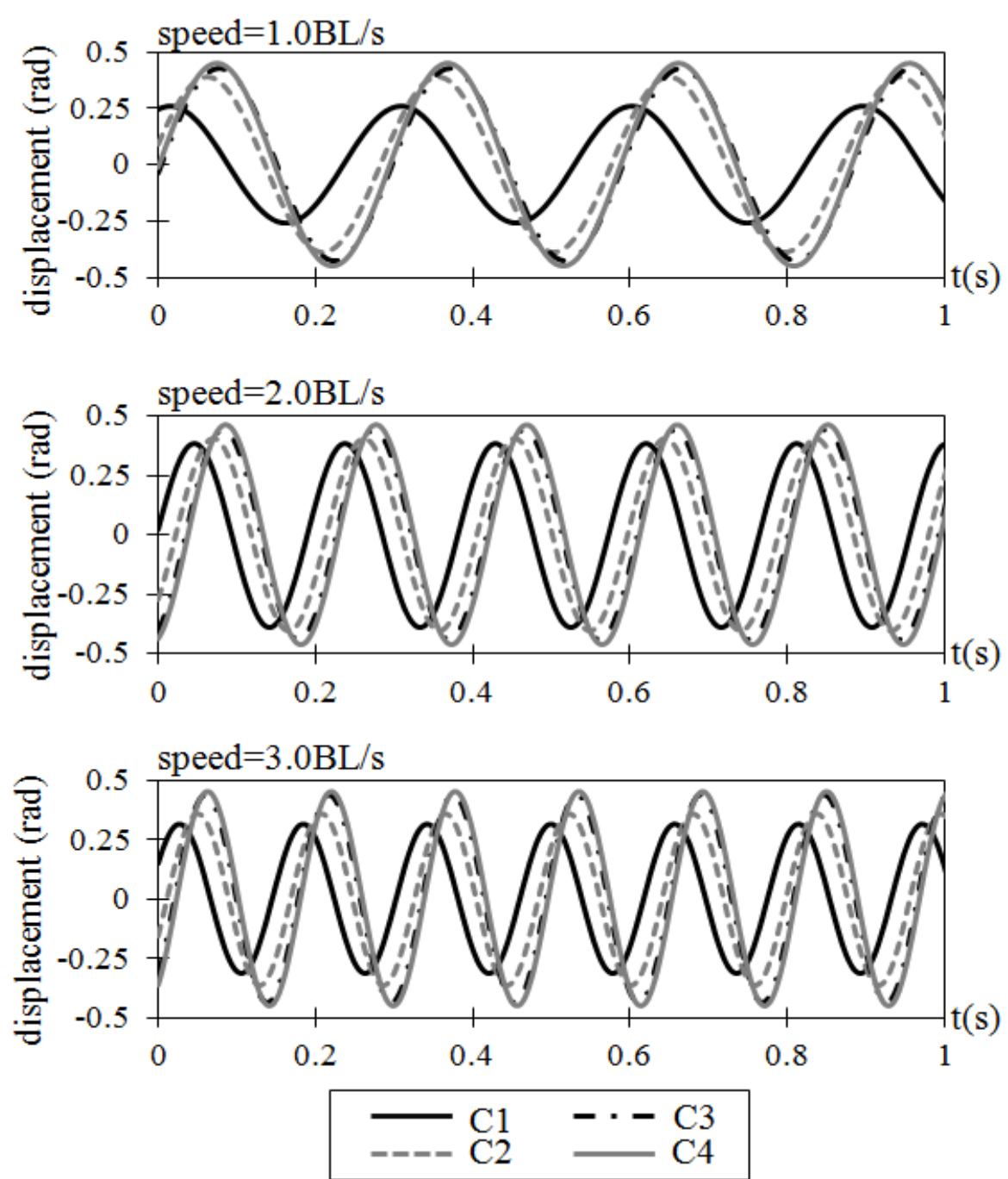

Fig. 6 Displacement of points $\mathrm{C} 1, \mathrm{C} 2, \mathrm{C} 3$ and $\mathrm{C} 4$ at velocities of 1.0, 2.0 and 3.0 BL/s. 


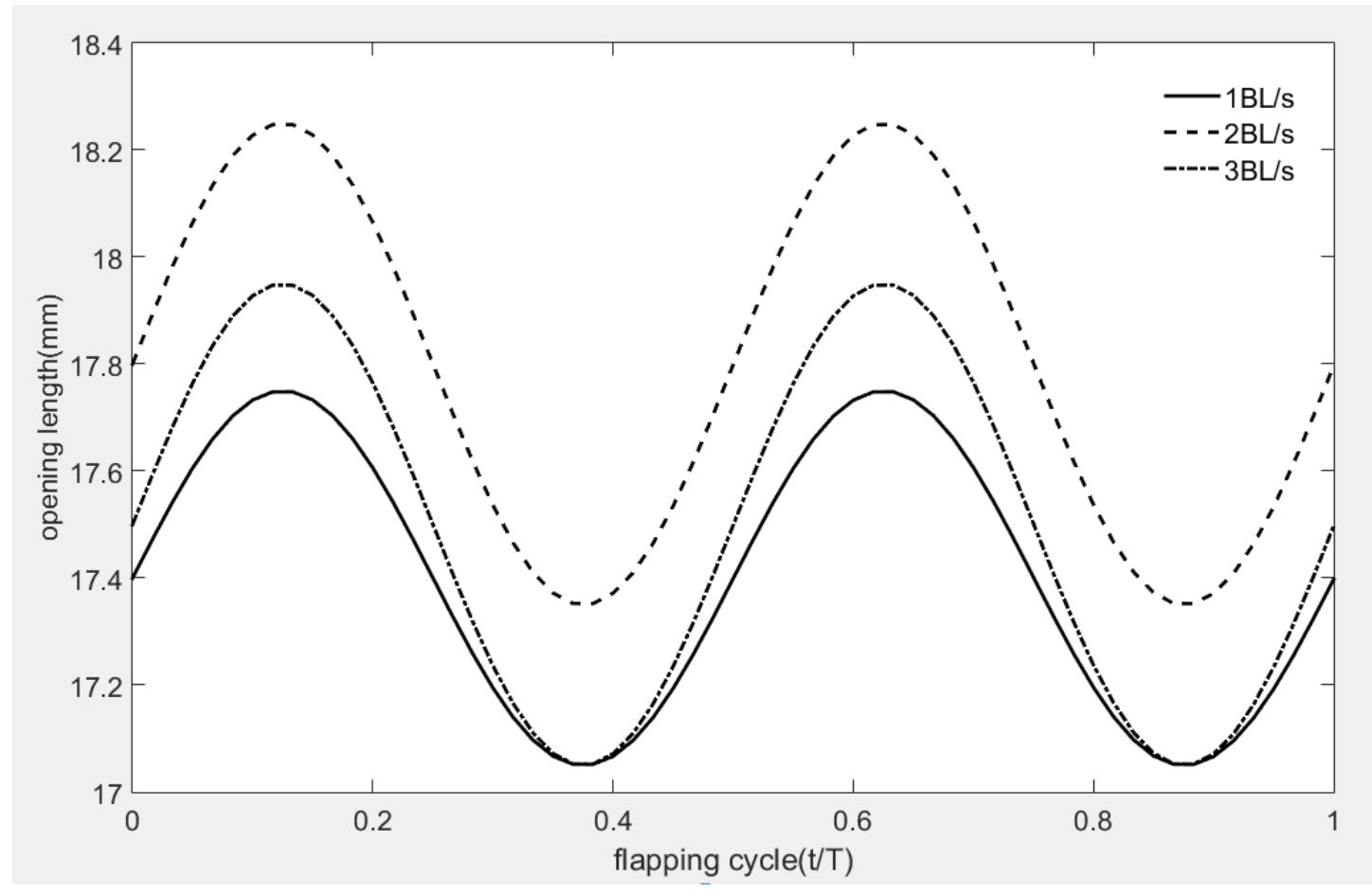

Fig. 7 Opening length of caudal at velocities of 1.0, 2.0 and 3.0 BL/s 

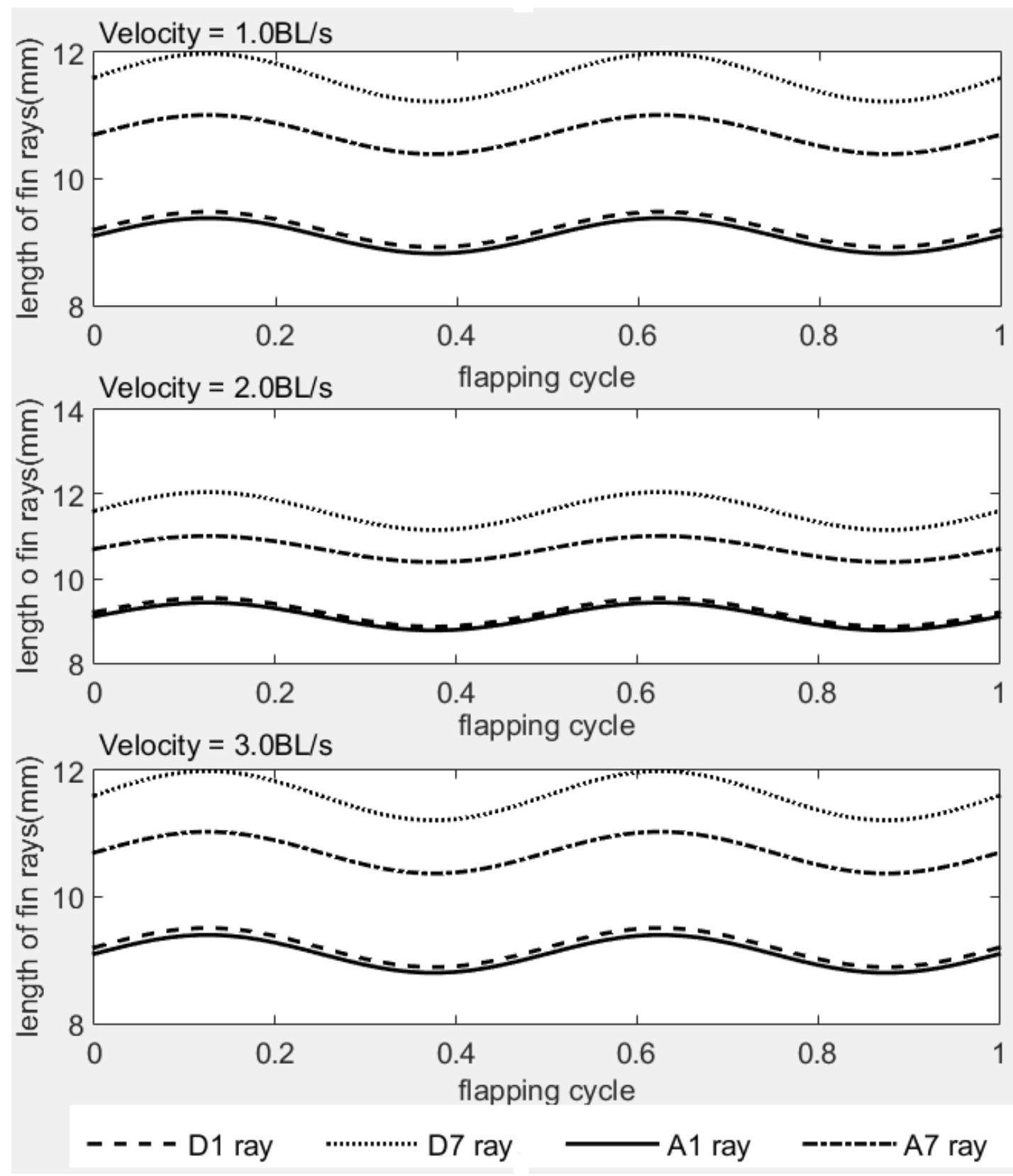

Fig. 8 Variation of fin rays in one oscillating cycle 


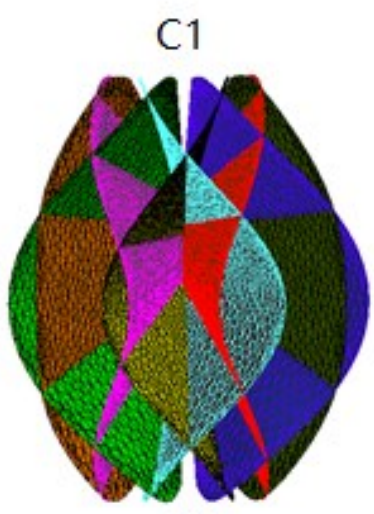

$\mathrm{C} 7$

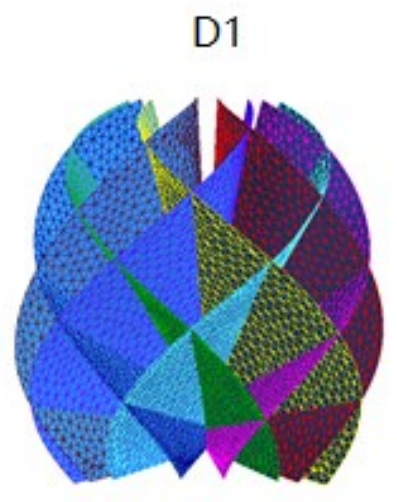

D7

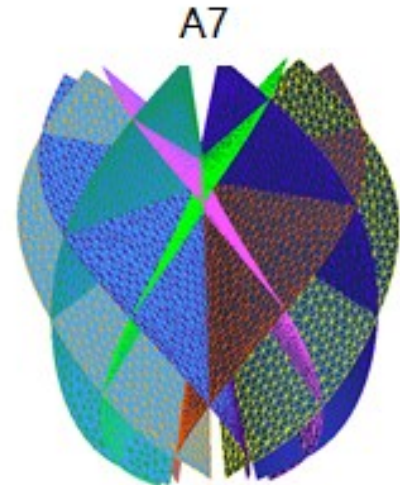

A1

caudal

dorsal

anal

Fig. 9 Deformations and envelopes in one beat cycle of caudal, dorsal and anal fins. Different colors shows different time instances. $\mathrm{C} 1$ and $\mathrm{C} 7$ rays are leading-edges for caudal; D1 and D7 rays are leading-edges and trailing-edges for dorsal, respectively; A1 and A7 rays are leading-edges and trailing-edges for anal, respectively. 

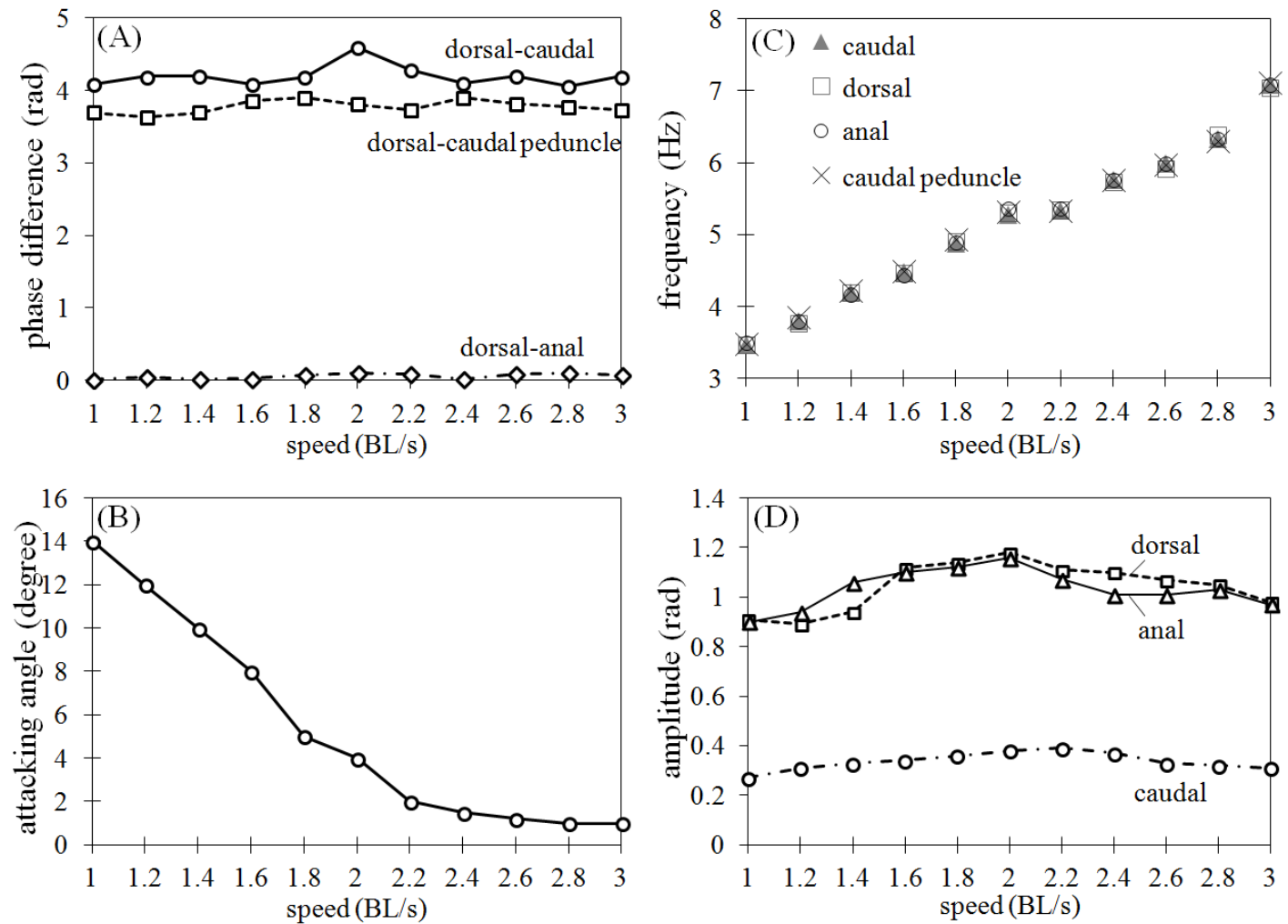

Fig. 10 (A) Phase differences vs. swimming velocity among caudal, dorsal and anal fins, and between peduncle caudal and dorsal; (B) Heading angle vs swimming velocity; (C) Oscillating frequency vs swimming velocity; and (D) Amplitude vs swimming velocity. 


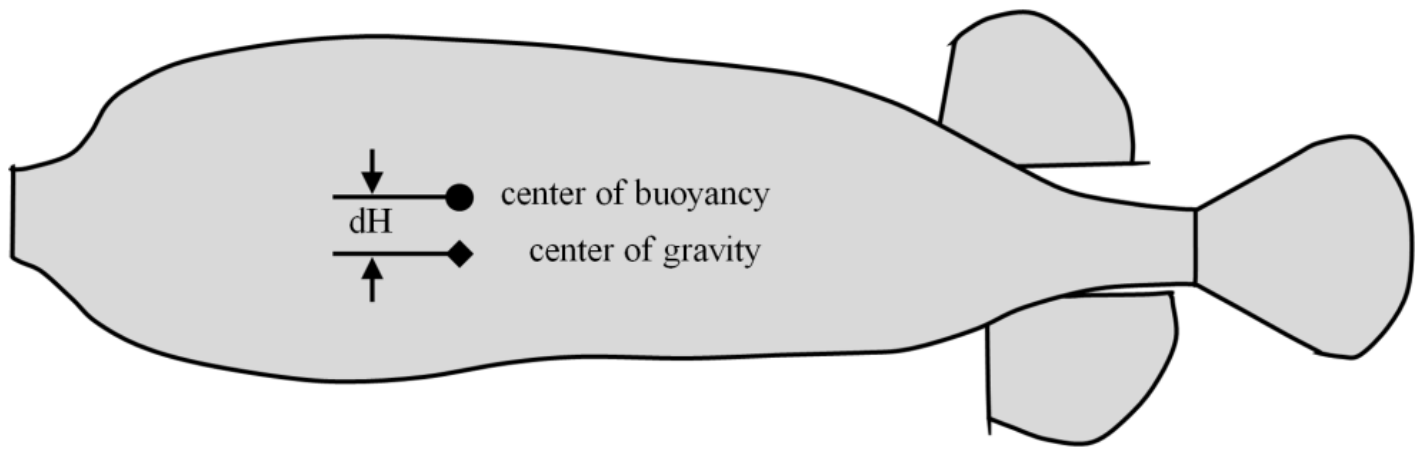

Fig. 11 A CFD model of pufferfish with a body and caudal, dorsal and anal fins. The dot shows the center of buoyancy (centroid of the fish body); the diamond represents the center of mass; $\mathrm{dH}$ represents the distance between centers of buoyancy and mass. In simulation, $\mathrm{dH}$ was set respectively to $0.00(\mathrm{CM} 1), 0.02(\mathrm{CM} 2), 0.04$ (CM3) of BL. The swimming direction is in accordance with $\mathrm{x}$-direction; and the gravitational direction is in the opposite direction of y-axis. 


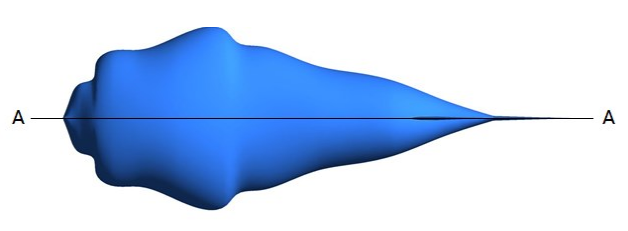

(a) Vertical section

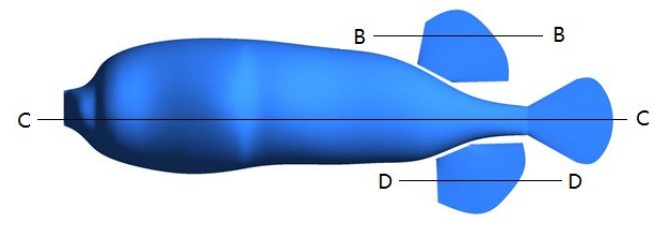

(b) Horizontal sections

Fig. 12 AA-plane is neutral surface in vertical section; BB-plane, CC-plane, and DD-plane are horizontal sections of dorsal, caudal and anal fins, respectively. 


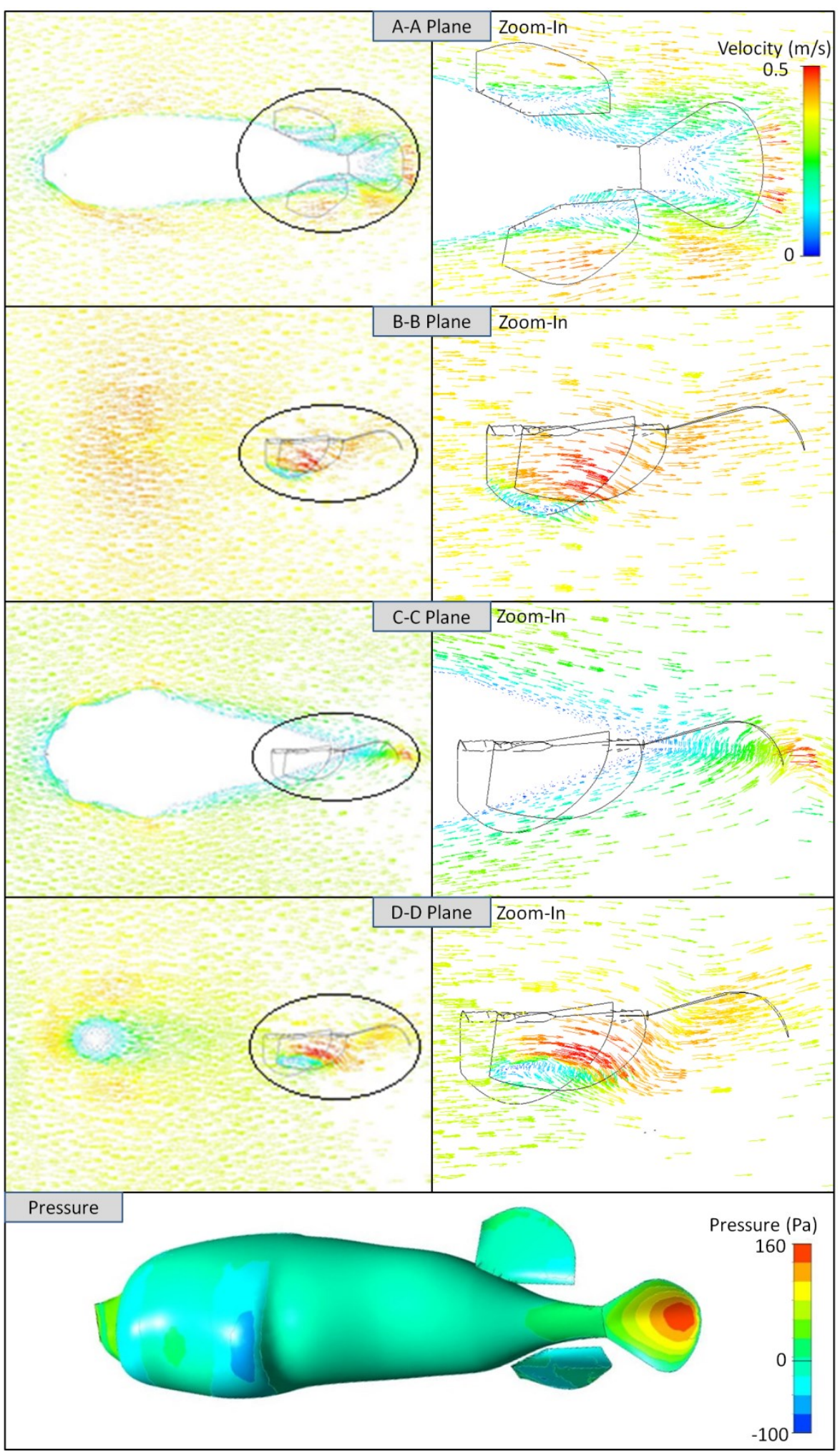

Fig. 13 Velocity vectors (A-A, B-B, C-C and D-D Plane) and pressure distributions on body and fin surfaces at $3.0 \mathrm{BL} / \mathrm{s}$ 


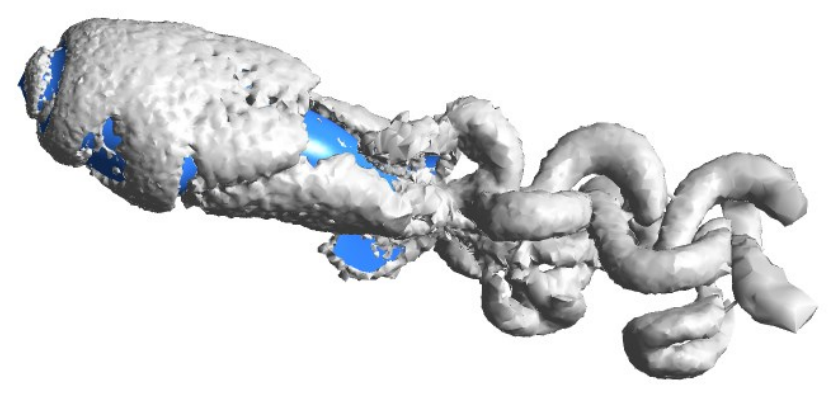

\section{$\mathrm{A} \quad 1.0 \mathrm{BL} / \mathrm{s}$}

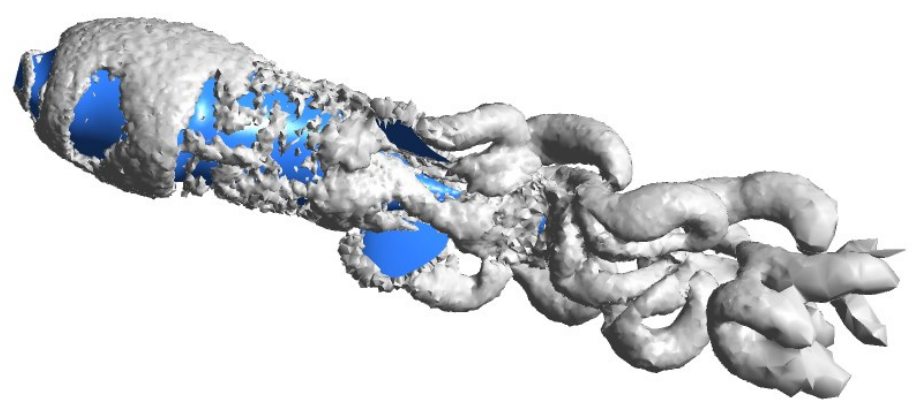

B $2.0 B L / s$

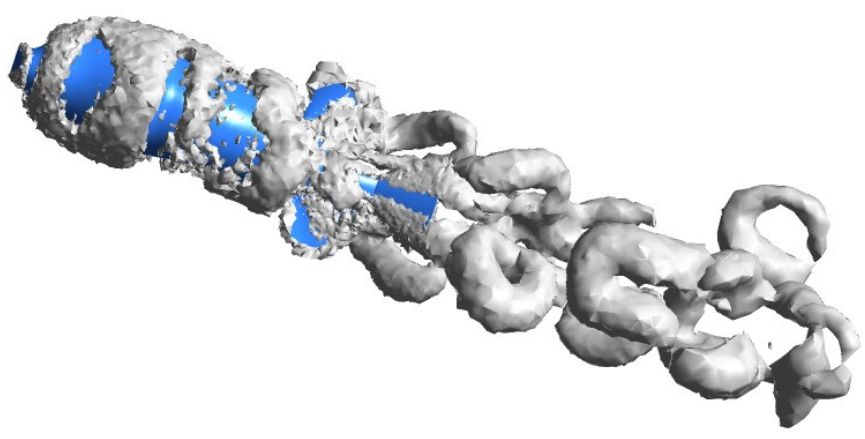

\section{3.0BL/s}

Fig. 14 Wake topology visualized in terms of iso-surfaces $\mathrm{Q}=0.02$ and 0.01 at swimming speeds of (A) 1.0 BL/s; (B) $2.0 \mathrm{BL} / \mathrm{s}$; (C) $3.0 \mathrm{BL} / \mathrm{s}$, respectively. 